\title{
SHORT COMMUNICATION Comments on Size Effects in Metallic Films
}

\author{
C. R. TELLIER and A. J. TOSSER \\ Universite de Nancy I, Laboratoire d'Electronique, C.O. 140, 54037 Nancy Cedex, France
}

(Received October 19, 1978)

In a recent paper $^{1}$ a new approximate expression for polycrystalline metal film resistivity has been compared to previously proposed expressions. ${ }^{1-4}$ We attempt in this paper to discuss the validity of the theoretical formulation.

The well-known Fuchs-Sondheimer (FS) conduction model $^{5}$ is applicable to thin films in which background and film surface scatterings occur. The film resistivity $\rho_{F}^{F S}$ is given by: ${ }^{5}$

$$
\rho_{F}^{F S}=\rho_{B} \cdot[F(k, p)]^{-1}
$$

where $\rho_{B}$ is the bulk resistivity (i.e. the resistivity of an infinitely thick film), $k$ the reduced thickness, $p$ is the reflection coefficient at the film surfaces and $F$ the usual size effect function or $F S$ function. (Refer to Warkusz ${ }^{1}$ (Eq. 2) and Sondheimer ${ }^{5}$ ).

Eq. (1) cannot be used to express the relation between the resistivity of a polycrystalline film $\rho_{F}^{M S}$ and the resistivity $\rho_{g}$ of an infinitely thick polycrystalline film in the Mayadas-Shatzkes model ${ }^{6}$ (when background, grain-boundary and surfaces scatterings occur simultaneously) since the background relaxation time $\tau$ of the $F-S$ model is replaced by a relaxation time $\tau^{*}$ representing the combined effect of background and grain-boundary scatterings (Refer to Mayadas and Shatzkes, ${ }^{6}$ Eq. (76)). To obtain the total film resistivity in presence of both background, grain-boundary and surface scatterings, the relaxation time $\tau^{*}$ is then substituted for $\tau$ in the Boltzmann equation and in relations deduced from boundary conditions at film surfaces; hence, the obtained relation between $\rho_{F}^{M S}$ and $\rho_{g}$ (Mayadas and Shatzkes ${ }^{6}$ Eq. (15) and Mola and Heras ${ }^{7}$ ) cannot be reduced to Eq. (1).

Consequently, all relations related to polycrystalline films which could be deduced from the following incorrect relation: ${ }^{1}$

$$
\rho_{F}^{M S}=\rho_{g} \cdot[F(k, p)]^{-1}
$$

are questionable. This is the case for Eq. (6) of Warkusz ${ }^{1}$ and all derived equations. For large ranges of $k, p$ and the physical parameter $\alpha^{6}$ (specially in the usual thin films ranges); it is observed, ${ }^{1}$ as expected, that this formulation markedly deviates from the theoretical Mayadas-Shatzkes $(M S)$ relation. However no marked discrepancy is observed when $k \gg 1$ since the physical effect arising because of the geometrical limitation of the mean free path imposed by the film surfaces vanishes, i.e.

$$
\rho_{F}^{M S} / \rho_{g} \approx 1
$$

It has been shown ${ }^{8-15}$ that several methods exist to reduce $M S$ expressions to analytical expressions and specially to Fuchs-Sondheimer expressions. ${ }^{10,11,14}$ For instance an effective mean free path may be introduced, leading to a substitution of $k$ by $k_{g}$ where $k_{g}$ is defined by:

$$
k_{g}=k \cdot[G(\alpha)]^{-1}
$$

with $G(\alpha)=\rho_{0} / \rho_{g}$ where $\rho_{0}$ is the resistivity of a single crystal, i.e. the resistivity that refers to background scattering. Hence, convenient expressions for $\rho_{F}^{M S}$ could be ${ }^{10,11,14}$

$$
\begin{aligned}
& \rho_{F}^{M S} / \rho_{g}=\left[F\left(k_{g}, p\right)\right]^{-1} ; \\
& \rho_{F}^{M S} / \rho_{0}=\left[F\left(k_{g}, p\right)\right]^{-1} \cdot[G(\alpha)]^{-1}
\end{aligned}
$$


In the limit when $k_{g}$ becomes large $;^{12}$

$$
\rho_{F}^{M S} / \rho_{g} \approx 1+(1-p) k^{-1} \cdot \frac{3}{8} G(\alpha)
$$

It has been previously shown ${ }^{4}$ that an approximate expression for $\rho_{F}^{M S}$ could be:

$$
\rho_{F}^{M S} / \rho_{g} \approx 1+(1-p) k^{-1} \cdot h(\alpha)
$$

In the limiting $k_{g}$-range $\left(k_{g} \gg 1\right)$ it appears that:

$$
h(\alpha) \approx \frac{3}{8} G(\alpha)
$$

Numerical data ${ }^{4}$ have shown that the following relation

$$
h(\alpha)=\frac{3}{8} \cdot G(\alpha)
$$

proposed by Warkusz ${ }^{1}$ leads to more significant deviation when we consider large $\alpha, p$ and $k$ ranges than the model previously derived by Tellier and Tosser. ${ }^{4}$
Hence, we may conclude from the above discussions that the conduction model proposed by Warkusz is questionable.

\section{REFERENCES}

1. F. Warkusz, Electrocomp. Sci \& Technol., 5, 99 (1978).

2. P. Wissman, Thin Solid Films, 5, 329 (1970).

3. F. Thieme and W. Kirstein, Thin Solid Films, 30, 371 (1975).

4. C. R. Tellier and A. J. Tosser, Thin Solid Films, 33, L 19 (1976).

5. E. H. Sondheimer, $A d v$. Phys., 1, 1 (1952).

6. A. F. Mayadas and M. Shatzkes, Phys. Rev. B, 1, 1382 (1970).

7. E. E. Mola and J. M. Heras, Thin Solid Films, 18, 137 (1973).

8. C. R. Tellier, Electrocomp. Sci \& Technol., 5, 127 (1.978).

9. C. Tellier and A. Tosser, Le Vide, Suppl., 189, 25 (1978).

10. C. Tellier and A. Tosser, Le Vide, 189, 129 (1977).

11. C. R. Tellier, A. J. Tosser, and C. Boutrit, Thin Solid Films, 44, 201 (1977).

12. C. R. Tellier, Thèse Doctorat ès Sciences, Nancy (1977).

13. C. R. Tellier and A. J. Tosser, Thin Solid Films, 43, 261 (1977).

14. C. R. Tellier, Thin Solid Films, 51, 311 (1978).

15. C. R. Tellier and A. J. Tosser, Int. Conf. Thin Films, Loughborough G.B. (1978) Thin Solid Films, 57, 163 (1979). 

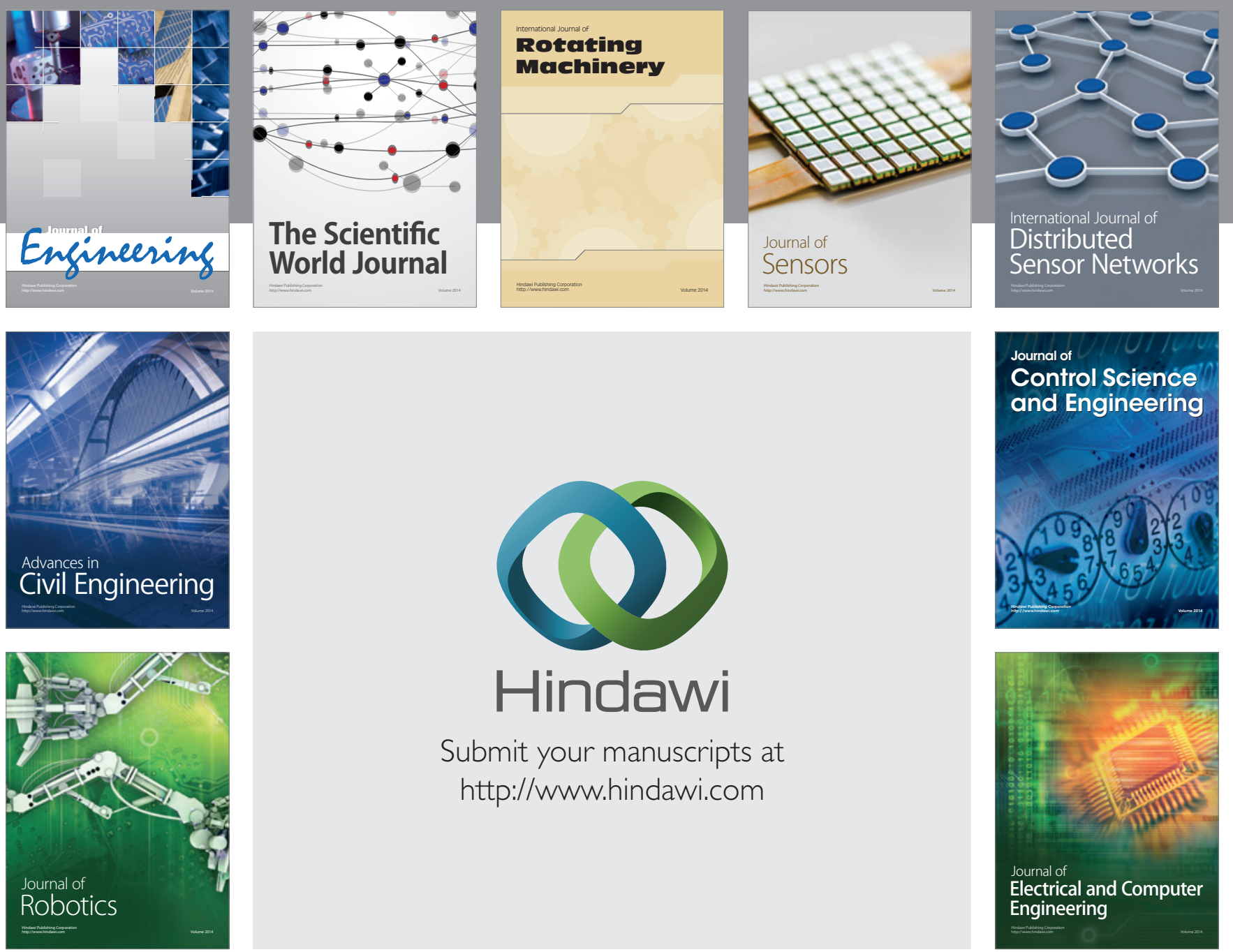

Submit your manuscripts at

http://www.hindawi.com
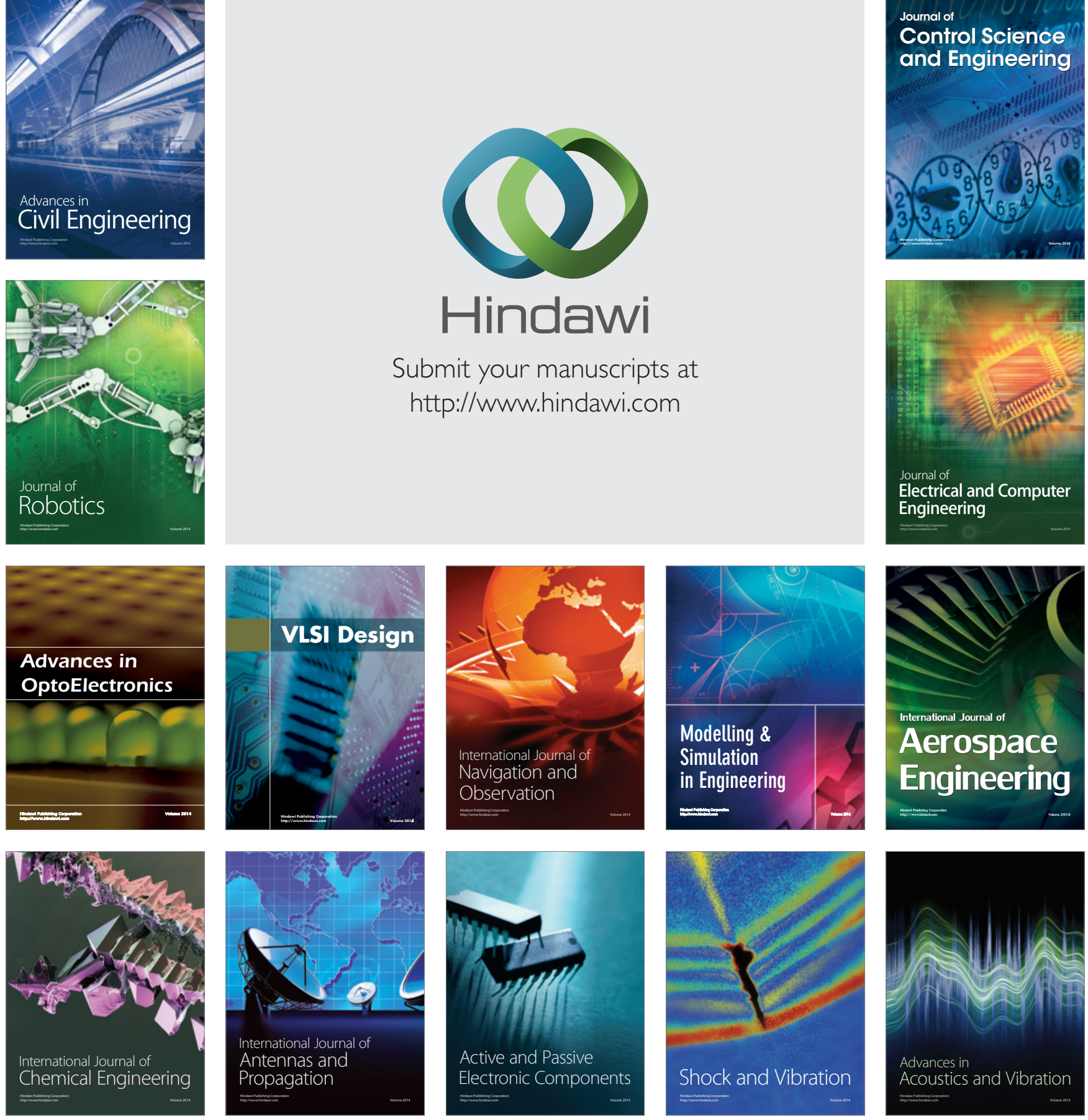